\title{
Malária em Minas Gerais, Brasil, no Período 1980-1992
}

\author{
Malaria in the State of Minas Gerais, Brazil, 1980-1992
}

Kátia Maria Chaves ${ }^{1}$; José Francisco Zumpano ; Marcelo C. de Resende $^{1}$;
Fabiano G. Pimenta Junior ${ }^{1}$ \& Manoel Otávio C. Rocha ${ }^{2}$

CHAVES, K. M.; ZUMPANO, J. F.; RESENDE, M. C.; PIMENTA JR., F. G.; ROCHA, M. O. C. Malaria in the State of Minas Gerais, Brazil, 1980-1992. Cad. Saúde Públ., Rio de Janeiro, 11 (4): 621-623, Oct/Dec, 1995.

Epidemiological analysis of malaria in the State of Minas Gerais, Brazil, shows the need for permanent reporting of the endemic-related data in order to maintain clinical suspicion of the infection in any febrile patient. Moreover, it increases the possibility of early aetiological diagnosis. Such reporting aims at improving performance of the local health system. It is important to maintain a well-organized malaria epidemiological surveillance system even in non-endemic areas.

Key words: Malaria; Plasmodium sp.; Epidemiology; Malaria Control

\section{INTRODUÇÃO}

A existência de áreas de alta transmissão de malária na Amazônia Legal constitui importante problema de saúde pública, influenciando não apenas áreas vizinhas, como também exercendo pressão sobre vários Estados brasileiros. O fluxo migratório de pessoas entre Minas Gerais e as áreas endêmicas da malaria, associado à presença de áreas consideradas malarígenas no estado, são fatores que favorecem a reintrodução e a disseminação local da doença (MS/Sucam/DRMG, 1990). A Fundação Nacional de Saúde em Minas Gerais (FNS/MG) tem procurado acompanhar sistematicamente o comportamento regional da malária, atuando através da busca de portadores da parasitose, do diagnóstico e tratamento dos doentes e do controle de focos. O presente trabalho tem como objetivo descrever aspectos particulares da ocorrência de malária em Minas Gerais, no período de 1980 a 1992, com ênfase para o ano de 1992.

\footnotetext{
${ }^{\prime}$ Fundação Nacional de Saúde. Avenida Brasil 2023, 3- andar, Belo Horizonte, MG, 30140-002, Brasil

${ }^{2}$ Grupo de Estudo e Controle da Malária. Faculdade de Medicina, Universidade Federal de Minas Gerais. Avenida Professor Alfredo Balena. 190, sala 3057, Belo Horizonte, MG, 30130-100, Brasil.
}

\section{MATERIAL E MÉTODOS}

Foram analisadas duas amostras. A primeira correspondia aos casos notificados de malária em Minas Gerais no ano de 1992 e, a segunda, aos registros de focos autóctones no estado, no período de 1980 a 1992. Os dados foram obtidos a partir de informações arquivadas no setor de estatística da FNS/MG.

Foi estudada a freqüência dos casos por: origem, idade, sexo, atividade profissional dos pacientes (ligada ao contágio), agente etiológico, história anterior de malária, relato de internação recente, ocorrência de óbito, verificação de cura e mês de atendimento dos pacientes. Quanto aos registros autóctones, foi estudada a frequiência dos casos e o número de focos.

\section{RESULTADOS}

O número de pacientes com malária atendidos no Brasil e em Minas Gerais, no período de 1980 a 1991, encontra-se exposto na Tabela 1. Observa-se que o percentual de lâminas positivas para malária, em Minas Gerais, é baixo em relação ao País. Nos anos de 1985 e 1986, contudo, ao contrário do que ocorreu no Brasil, Minas Gerais apresentou aumento de registros.

Em 1992 foram colhidas amostras de sangue (gota espessa) de 45.306 pacientes com suspei- 
TABELA 1. Lâminas Positivas de Malária no Brasil e em Minas Gerais - 1980 a 1991

\begin{tabular}{|c|c|c|c|c|}
\hline \multirow[b]{2}{*}{ Ano } & \multirow{2}{*}{$\frac{\text { Brasil }}{\mathrm{N}^{\mathrm{o}}}$} & \multicolumn{2}{|c|}{ Minas Gerais } & \multirow[b]{2}{*}{ Casos Autóctones } \\
\hline & & $\mathrm{N}^{\mathrm{o}}$ & $\%$ & \\
\hline 1980 & 169.871 & 287 & 0,17 & 70 \\
\hline 1981 & 197.149 & 303 & 0,15 & 6 \\
\hline 1982 & 221.969 & 408 & 0,18 & 0 \\
\hline 1983 & 297.687 & 646 & 0,22 & 31 \\
\hline 1984 & 505.257 & 809 & 0,16 & 23 \\
\hline 1985 & 399.462 & 1.061 & 0,27 & 5 \\
\hline 1986 & 443.627 & 1.142 & 0,26 & 31 \\
\hline 1987 & 508.864 & 979 & 0,19 & 9 \\
\hline 1988 & 559.535 & 880 & 0,16 & 43 \\
\hline 1989 & 577.520 & 994 & 0,18 & 201 \\
\hline 1990 & 560.396 & 647 & 0,11 & 28 \\
\hline $1991^{(\dagger)}$ & 541.396 & 435 & 0,08 & 14 \\
\hline
\end{tabular}

ta da doença. Foi confirmada a presença de Plasmodium em 325 lâminas, o que representou uma redução de 110 casos, ou seja, 25,3\% em relação ao ano anterior.

A média mensal de atendimentos no ano de 1992 foi de 27 pacientes. As maiores frequiências ocorreram nos meses de agosto, setembro e fevereiro, correspondendo a, respectivamente, 42 $(13,0 \%), 40(12,3 \%)$ e $38(11,6 \%)$ registros.

Dos 325 pacientes com malária detectados em Minas Gerais, no ano de 1992, a maioria $(93,0)$ correspondeu a casos importados, especialmente da Região Amazônica. Do total de 298 registros procedentes desta área, destacam-se aqueles dos Estados do Mato Grosso (119 casos - 36,6\%), Rondônia (95 casos - 29,2\%) e Pará (53 casos $-19,3 \%)$.

Dez casos autóctones foram detectados em Minas Gerais em 1992, os quais se originaram nos seguintes municípios: Governador Valada- res e Açucena (dois casos); Araguari e Tupaciguara (cinco casos) e São Francisco (três casos). Todos estes municípios encontram-se situados em áreas consideradas maláricas pela FNS/MG.

A idade dos pacientes variou de quatro meses a 72 anos (média de 32 anos), tendo os grupos etários entre 21 a 35 anos e 36 a 50 anos contribuído com a maior concentração dos registros.

Verificou-se amplo predomínio de acometimento do sexo masculino, com 282 casos $(81,0 \%)$. Quanto à profissão e/ou atividade dos pacientes, observou-se $26,0 \%$ de garimpeiros, $10,0 \%$ de lavradores e $5,2 \%$ de domésticas. Sessenta e seis doentes $(20,0 \%)$ informaram terem realizado viagem de lazer a áreas malarígenas, sendo a pesca o maior motivo de deslocamento.

Dos 325 casos estudados em 1992, 187 $(57,5 \%)$ informaram a ocorrência de episódios 
anteriores de malária. Cinqüenta e seis pacientes relataram história de internação hospitalar prévia em decorrência de malária. Duzentos e trinta e sete $(73,0 \%)$ não compareceram à rotina de verificação de cura e apenas um óbito foi registrado.

Observou-se predomínio de infecção por Plasmodium vivax (238 casos, $73,0 \%$ ) quando comparada ao número daquelas por Plasmodium falciparum (81 casos, $25,0 \%$ ) e por infecção mista (seis casos, 2,0\%).

No período de 1980 a 1992, detectaram-se 29 focos de malária no estado de Minas Gerais, que foram responsáveis pelo surgimento de $471 \mathrm{ca}$ sos autóctones.

\section{DISCUSSÃO}

O estado de Minas Gerais apresenta área de $586.624,3 \mathrm{~km}^{2}$, encontra-se situado no sudeste do Brasil, possuindo área malárica de $384.431,3 \mathrm{~km}^{2}$. Há vários anos registra poucos casos de transmissão local, sendo a maioria dos pacientes procedente de estados situados em área endêmica.

Em 1985 e 1986 foi observado aumento de notificação por malária no estado, a maioria composta por casos importados da Amazônia. Acredita-se que a circulação local de garimpeiros oriundos da Região Amazônica seja o fator responsável pela observação do fenômeno (MS/ Sucam/DRMG, I 981).

A partir de 1990, nota-se diminuição dos casos notificados em Minas Gerais, provavelmente devido à crise sócio-econômica do país, com conseqüente redução das viagens de lazer às áreas hiperendêmicas, geralmente distantes, e às cidades de origem dos migrantes, para visitas familiares. $\mathrm{O}$ alto percentual de pacientes que não compareceram à rotina de verificação de cura pode ser atribuído ao fato de que grande parte dos doentes não permanece em Minas Gerais, encontrando-se apenas em trânsito.

Apesar da ocorrência anual de casos autóctones ser pequena em Minas Gerais, quando comparada às áreas hiperendêmicas do país, é importante o acompanhamento epidemiológico e o controle destes focos, pois os mesmos podem possibilitar a reintrodução da doença no estado.

\section{RESUMO}

CHAVES, K. M.;ZUMPANO, J. F.; RESENDE, M. C.; PIMENTA JR., F. G.; ROCHA, M. O. C. Malária em Minas Gerais, Brasil, no Período 1980-1992. Cad. Saúde Públ., Rio de Janeiro, 11 (4): 621-623, out/dez, 1995.

A análise epidemiológica da malária em Minas Gerais mostra a necessidade de divulgação permanente da situação da transmissão da doença, de forma a contribuir para a suspeita clínica em casos febris e para o diagnóstico precoce. A divulgação das informações técnicas sobre a malária visa ao melhor desempenho dos serviços de saúde locais, a fim de controlar os casos detectados e diminuir as taxas de morbi-mortalidade. Considera-se ser importante a manutenção de sistema organizado e ativo de vigilância epidemiológica de malária, mesmo em áreas endêmicas.

Palavras-Chave: Malária Plasmodium sp.; Epidemiologia; Controle da Malária

\section{REFERÊNCIAS BIBLIOGRÁFICAS}

MS (Ministério da Saúde)/SUCAM (Superintendência de Campanhas de Saúde Pública)/DRMG (Diretoria Regional de Minas Gerais), 1981. Relatório Anual das Atividades do Programa de Controle da Malária em Minas Gerais no Ano de 1985. Relatório Interno, Belo Horizonte: Sucam/DRMG. (Mimeo.)

1990. A Malária em Minas Gerais. Informativo Epidemiológico. Belo Horizonte: Sucam/DRMG. (Mimeo.)

MS (Ministério da Saúde), 1992. Séries históricas de agravos e doenças transmissíveis; Malária-lâminas positivas diagnosticadas por U.F. Brasil, 1980 a 1991. Informe Epidemiológico do SUS, 1: 35-40. 